\title{
Uneven Social Policies: The Politics of Subnational Variation in Latin America
}

\author{
Sara Niedzwiecki
}

Cambridge University Press, 2018, 272 páginas

\section{Ismael Cassini}

Durante las últimas dos décadas, numerosos países de América Latina han sido el escenario de una fuerte expansión de políticas sociales, en particular, de los programas de transferencias condicionadas de ingreso (PTCI). Debido a las reglas de asignación bien definidas que los caracterizan, los PTCI han sido catalogados como políticas universalistas y no pasibles de ser utilizadas de forma clientelar. Sin embargo, cuando se deja de lado el diseño y se observa la implementación de las mismas, un nuevo interrogante sale a la luz: ¿Por qué estos programas universalistas no tienen un alcance territorialmente homogéneo? Uneven Social Policies, de Sara Niedzwiecki, responde a esta pregunta a través de un planteo teórico novedoso y un sólido trabajo empírico aplicado a Argentina y Brasil.

El trabajo de Niedzwiecki toma elementos de tres literaturas existentes en la disciplina. En primer lugar, inscribe a su objeto de estudio, los PTCI, como piezas claves en la evolución reciente del Estado de Bienestar en América Latina (Huber y Stephens 2012). En segunda instancia, la autora delimita el alcance de su teoría a los Estados multinivel, es decir, aquellos en los que conviven dos o más niveles de gobierno y cada uno de ellos tiene áreas de decisión autónomas y otras de decisión compartida (Hooghe et al. 2016). Finalmente, - y en línea con investigaciones recientes- el argumento del libro rescata una vieja discusión en torno a la atribución de responsabilidades y el retorno electoral de las políticas públicas (Zucco 2013).

Desde ese lugar, Niedzwiecki propone una parsimoniosa teoría basada en tres variables explicativas: el alineamiento político entre los diferentes niveles de gobierno (nacional, provincial/estadual y municipal), los legados de políticas y la infraestructura territorial. Si bien cada una de ellas es ingresada de forma aditiva en su modelo teórico, el gran aporte del libro radica en los hallazgos vinculados al alineamiento político.

Más precisamente, la autora afirma que existen dos tipos de políticas sociales: por un lado, están aquellas que poseen atribución de responsabilidades claras, es decir, que los beneficiarios de las mismas saben cuál es el nivel de gobierno responsable de su creación y, por ende, al que deberían retribuir al momento de votar. Por el otro, existen políticas sociales cuya atribución de responsabilidad es difusa ya que quienes reciben los beneficios no están seguros de cuál es el nivel de gobierno que las puso en marcha. Consecuentemente, la relevancia del alineamiento político como variable explicativa es condicional al tipo de política social que se analiza: sólo cuando la atribución de responsabilidades es clara, los gobernantes subnacionales tendrán expectativas de que su correcta implementación ayu- 
dará al gobierno nacional. Esto último generará incentivos en los oficialismos subnacionales no alienados con el gobierno nacional para obstruir dicha implementación. Mientras que los PTCI pertenecen al primer grupo, las políticas de salud orientadas a la mejora de la atención primaria se ubican dentro del segundo. A lo largo del trabajo, Niedzwiecki logra demostrar la existencia de esta asociación de tipo condicional a través de la comparación de los dos tipos de política.

La utilización de métodos mixtos también es destacable. La autora elabora estudios de casos anidados dentro de un análisis estadístico, tanto en Argentina como en Brasil. Mediante esta estrategia, el libro logra describir en detalle los mecanismos causales, sin perder capacidad de generalización de su propuesta teórica (validez externa). Por un lado, el análisis cuantitativo se desarrolla en forma de un estudio longitudinal utilizando una base de datos original. Por el otro, y de forma complementaria, se presentan estudios de casos comparados de PTCI y de programas de salud en dos países, Argentina y Brasil, cuatro provincias/estados (dos por país) y ocho municipalidades (dos por provincia/estado) construidos gracias a una impresionante cantidad de entrevistas.

En línea con su hipótesis condicional, la autora muestra que el alineamiento político es predictor del nivel de implementación de las políticas sociales solamente cuando se analizan la Asignación Universal por Hijo (AUH) y el Bolsa Familia. En estos casos, los oficialismos subnacionales obstaculizan la implementación de las políticas mediante tres acciones: compiten con la política nacional mediante programas sociales propios, montan obstáculos burocráticos y/o actúan por omisión no dándole información importante al nivel nacional. Por el contrario, cuando existe alineamiento entre niveles, las políticas provinciales y municipales funcionan como complemento de los PTCI nacionales.

Los legados de política, la segunda variable explicativa, son importantes tanto para los PTCI como para las políticas de salud orientadas a fortalecer la atención primaria. Sin embargo, estos legados no siempre operan de la misma manera. Mientras que la cobertura de la AUH y la de la Bolsa Familia se vieron favorecidas por la existencia de otras políticas de asistencia monetaria a familias pobres, el alcance de la Estratégia Saúde da Família en Brasil se vio fuertemente perjudicado por la presencia de actores organizados que fueron empoderados por las estrategias sanitarias adoptadas previamente.

De la misma manera, la infraestructura territorial es otro factor que influye sobre ambos tipos de políticas. Desagregada en dos dimensiones, esta variable incluye, por un lado, el alcance territorial de las instituciones estatales y, por el otro, la fortaleza de las relaciones entre la sociedad civil y el Estado. Cuanto más sólidas sean estas dos, mayor será el impacto positivo sobre el nivel de implementación de las políticas sociales. Asimismo, esta variable le permite a Niedzwiecki introducir el nivel de democracia subnacional como un factor explicativo indirecto en tanto éste se asocia positivamente con la fortaleza de la sinergia Estado-sociedad civil.

En el plano teórico, el aspecto más cuestionable del libro es la ausencia de costos electorales para los políticos subnacionales que obstruyen la implementación de las políticas sociales. Si bien la teoría de la autora se asienta en las 
expectativas de los agentes, los gobernantes subnacionales parecen nunca preocuparse por los posibles efectos negativos que sus acciones puedan tener en el plano electoral. En un sentido similar, el libro no profundiza en las estrategias que los gobiernos nacionales pueden desplegar como represalia a los obstáculos montados por sus pares de niveles inferiores.

A nivel metodológico, la selección de casos deja abierto otro interrogante: ¿qué pasa en provincias argentinas no alineadas y de bajos ingresos? Si bien la comparación de dos provincias de ingresos medios (San Luis y Mendoza) es una estrategia acertada para controlar por explicaciones basadas en la economía, no es del todo claro que el mecanismo causal sea el mismo en provincias de bajos recursos que no tienen los medios para competir con el Estado nacional a través de políticas sociales propias.

En síntesis, Uneven Social Policies es un notable ejemplo de cómo la economía política en conjunto con un enfoque multinivel de las instituciones estatales puede dar cuenta de los avances y nuevos desafíos que enfrenta el Estado de Bienestar en América Latina. A su vez, la teoría propuesta por Sara Niedzwiecki puede ser el puntapié inicial para futuros trabajos que intenten hacerla viajar a otros casos o bien a otras esferas de políticas. Finalmente, cabe mencionar que la relevancia sustantiva del trabajo de Niedzwiecki es enorme dado que pone en evidencia las condiciones que limitan el alcance de políticas sociales de mitigación de la pobreza en la región más desigual del mundo.

\section{Referencias bibliográficas}

Hooghe, L., G. Marks, A. Schakel, S. Niedzwiecki, S. Chapman Osterkatz y S. Shair-Rosenfield (2016). Measuring Regional Authority: A Postfunctionalist Theory of Governance, Volume I. Oxford: Oxford University Press.

Huber, E. y J. D. Stephens (2012). Democracy and the Left: Social Policy and Inequality in Latin America. Chicago: The University of Chicago Press.

Zucco, C. (2013). When Payouts Pay Off: Conditional Cash-Transfers and Voting Behavior in Brazil 20022010. En American Journal of Political Science 47 (3), 810-22. 